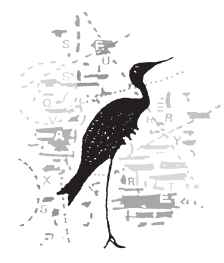

Guénaël Visentini

\title{
La psychanalyse : une science
}

\section{Introduction}

La question du lien entre psychanalyse et science se répète ad libitum dans le champ analytique. Si le fondateur de la psychanalyse en a exigé la scientificité, les générations d'analystes après lui ont pris la mesure de l'écart entre l'idéal qu'ils supposaient inhérent à « la » science et le statut difficile à saisir du savoir qu'ils produisaient. La psychanalyse : une science ? Ou pas.

\section{Spectroscopie d'un débat}

Il est prudent d'interroger d'abord la façon dont on pose la question. En effet, à suivre la littérature analytique dans ce débat, le mot science en jeu dans la question fait souvent référence, au prix de préjugés non fondés scientifiquement, à une forme de pensée idéalisée ou, à l'inverse, disqualifiée parce que jugée réductionniste. Deux types de réponse tournent ainsi en boucle : soit position rare de nos jours - la psychanalyse est dite «scientifique » et se pose alors le problème des critères permettant de le justifier ; soit le titre de scientificité est refusé, en une promotion - contre le positivisme - de l'âme (Jung), du parlêtre (Lacan) ou du sujet (large spectre de courants analytiques).

Ce maigre choix d'options quant au savoir psychanalytique - science impossible, anti-science - fait étonnamment l'impasse sur la pensée épistémologique freudienne et ses réflexions sur le statut du savoir scientifique, avec pour effet d'appauvrir le débat. Car, une fois la science écartée pour défaut de critères de scientificité, et puisqu'il ne reste plus rien pour penser le statut épistémologique de la psychanalyse - serait-elle une mythologie, une religion, de la littérature, de l'art, quoi d'autre encore ? -, l'on finit par se rabattre sur des options d'ordre idéologique : doit-on défendre la psychanalyse ? Est-elle un mal ou un bien ? Et les analystes de promouvoir l'analyse comme une 
expérience inclassable, salvatrice, subversive, qui permettrait à l'homme de retrouver ce que « la » science lui aurait ravi : sa parole, son désir, sa singularité, son inconscient.

Le but de cet article est de soutenir, en prenant appui sur Freud, une tout

1. Cette position a depuis lors été affinée et développée dans Pourquoi la psychanalyse est une science. Freud épistémologue, Paris, Puf, 2015.

2. Correspondance de Sigmund Freud avec le pasteur Pfister. 1909-1939, Paris, Gallimard, 1966, p. 124.

3. L'introduction de la psychanalyse aux États-Unis : autour de James Jackson Putnam, Paris, Gallimard, 1978, p. 200.

4. G. Bachelard, La formation de l'esprit scientifique. Contribution à une psychanalyse de la connaissance, Paris, Vrin, 2004 ; G. Canguilhem, Idéologie et rationalité dans l'histoire des sciences de la vie, Paris, Vrin, 2009 ; T. Kuhn, La structure des révolutions scientifiques, Paris, Flammarion, 1983 ; A.F. Chalmers, Qu'est-ce que la science? Popper, Kuhn, Lakatos, Feyerabend, Paris, Biblio essais, 1990 ; J.-M. Berthelot, L'emprise du vrai. Connaissance scientifique et modernité, Paris, Puf, 2008.

5. B. Latour, La science en action. Introduction à la sociologie des sciences, Paris, La Découverte, 2005 ; I. Stengers, L'invention des sciences modernes, Paris, Flammarion, 2011.

6. D. Bloor, Sociologie de la logique. Les limites de l'épistémologie, Paris, Pandore, 1983 ; M. Dubois, La nouvelle sociologie des sciences, Paris, Puf, 2001 ; T. Shinn et P. Ragouet, Controverses sur la science : pour une sociologie transversaliste de l'activité scientifique, Paris, Liber, 2005.

7. A. Prochiantz, Géométries du vivant, Paris, Collège de France/Fayard, 2008, p. 39-42 ; S. Firestein, Les continents de l'ignorance, Paris, Odile Jacob, 2014. autre position ${ }^{1}$.

\section{La question oubliée de la vérité}

Ainsi rapporté, le débat est très en dessous des exigences épistémologiques auxquelles Freud avait tenté de le porter. De son temps déjà, la psychanalyse était soutenue ou contestée à partir de perspectives axiologiques. Dans une lettre au pasteur Pfister, il s'en plaint :

«La satisfaction personnelle que l'analyse peut procurer, j'en ai joui au temps où j'étais seul ; et depuis que d'autres se sont joints à moi, j'ai eu plus de soucis que de joies. La manière dont les gens admettent l'analyse et l'utilisent ne m'a pas donné d'eux une autre opinion que leur comportement, quand ils la récusaient sans la comprendre ${ }^{2}$.»

Le geste épistémologique freudien, que nous proposons de redécouvrir, fut de réarticuler le problème en mettant au centre de la réflexion la question de la vérité, cette valeur pas toute idéologique, car s'y mêle la question d'un au-delà du discours - nommément, le « réel ».

L'idée, ici, est de donner un aperçu de cette position, « éthique [de] la vérité $^{3} »$ soutenant un scientifisme non scientiste, que les travaux les plus en pointe en épistémologie (Bachelard, Canguilhem, Kuhn, Chalmers, Berthelot ${ }^{4}$ ), en anthropologie des sciences (Latour, Stengers ${ }^{5}$ ), en sociologie des sciences (Bloor, Dubois, Shinn et Ragouet ${ }^{6}$ ), et que les témoignages des scientifiques actuels à propos de leurs pratiques (Alain Prochiantz, Stuart Firestein ${ }^{7}$ ) rejoignent par des voies diverses.

Nous proposons, dans un premier temps, de donner quelques indications sur le rapport complexe de Freud à la science, qui conduit - au-delà de la vulgate commune - à sa désidéalisation et à sa réouverture théorique. Puis nous verrons en quoi, pour Freud, la psychanalyse - comme théorie métapsychologique - est de fait une extension de la science à de nouveaux objets et devrait être considérée comme telle par les analystes.

\section{Freud et « la » science}

\section{La redéfinition freudienne}

Laplanche affirme qu' « il y a chez Freud certains fragments d'épistémo$\operatorname{logie} e^{8} \gg$. En effet, Freud n'a eu de cesse, au cours de son œuvre, de réfléchir à la nature du savoir qu'il produisait. De formation scientifique, c'est le statut de la science qu'il a questionné sans cesse. Quatre passages doivent ici être retenus :

«Ce n'est pas la nature différente des objets d'étude, mais la rigueur plus grande de la méthode utilisée lors de l'établissement des faits, ainsi que l'ambition de trouver une cohérence étendue qui constituent le caractère essentiel du travail scientifique ${ }^{9}$.» 
« Ce que nous voulons, à quoi l'on tend généralement dans la science, c'est une compréhension des phénomènes, l'instauration d'une corrélation entre eux et, en tout dernier lieu, là où c'est possible, un élargissement de notre pouvoir sur eux ${ }^{10}$.»

«La pensée scientifique n'est pas distincte, dans son essence, de l'activité de pensée normale que nous utilisons tous [...] quand nous prenons soin de nos affaires dans la vie. Elle n'a pris une forme particulière que par quelques aspects ; elle s'intéresse aussi à des choses qui n'ont pas d'utilité immédiate, tangible ; elle s'efforce de tenir soigneusement à distance les facteurs individuels et les influences affectives ; elle contrôle avec plus de rigueur, quant à leur fiabilité, les perceptions sensorielles sur lesquelles elle édifie ses conclusions ; elle se crée de nouvelles perceptions qu'il n'est pas possible d'atteindre par les moyens de tous les jours et isole les conditions de ces nouvelles expériences par des expérimentations intentionnellement variées. Elle aspire à atteindre la concordance avec la réalité, c'est-à-dire avec ce qui existe en dehors de nous, indépendamment de nous [...]. Cette concordance avec le monde extérieur, nous l'appelons vérité ${ }^{11}$.»

« Dans le travail scientifique, il est plus prometteur de s'attaquer à ce qui se trouve juste devant soi [...]. Si on le fait en allant bien au fond des choses, sans attente ni présupposition [...], il peut se faire, par suite de l'interdépendance qui rattache tout à tout, même le petit au grand, que [...] d'un travail [...] dénué de prétention, se dégage l'accès à l'étude des grands problèmes ${ }^{12}$. »

Retenons quatre idées de ces textes :

1. La science se définit par un travail psychique d'élaboration, non par les résultats de ce travail (lesquels dépendent aussi de la nature de l'objet).

2. La science propose un mode d'intelligibilité des phénomènes, en vue de pouvoir opérer sur eux au mieux.

3. La science n'est qu'une prolongation par d'autres moyens - dispositifs et méthodes réfléchis et contrôlés - de l'effort de vérité référentielle fait par la pensée quotidienne pour opérer sur le monde.

4. Tout phénomène peut être l'objet d'un travail d'investigation théoricoclinique et donc de résultats scientifiques.

La définition freudienne de la science la distingue ainsi clairement des autres savoirs : ses vérités sont produites à partir de dispositifs pensés pour serrer une logique empirique de l'objet (à la différence des vérités mythologiques, religieuses, philosophiques, littéraires ou artistiques, produites à partir de dispositifs autres, tels que la divination, la révélation, l'intuition ou l'inspiration). Par ailleurs, elles se distinguent des vérités de l'expérience quotidienne, par ce que Bachelard appellera une « rupture épistémologique » : l'expérience y est contrôlée en expérimentation ; les dispositifs et méthodes sont réfléchis au point que tout, du dispositif aux vérités produites, est critiquable par des tiers, au nom du désir commun de vérité, et au-delà de valeurs telles que le beau, le bien, le bon ou l'utile :

«Si un physicien peut vous prouver que la vie organique sur cette terre devra, à brève échéance, céder la place à une totale congélation, oserez-vous lui rétorquer à lui aussi : cela ne se peut pas ; cette perspective est trop peu réjouissante ? Je pense que vous vous tairez jusqu'à ce qu'un autre physicien vienne démontrer au premier qu'il a fait une erreur dans ses présuppositions ou ses calculs ${ }^{13}$.»

Ce qui relie les sciences entre elles, c'est le désir de vérité scientifique qui les fonde, ce désir de reconnaître « ce qui existe en dehors de nous, indépendamment de nous ». Il s'agit bien là d'une valeur, mais dont les spécificités sont à reconnaître. Du point de vue de la vérité, les autres types de savoir restent
Psychanalyse et science : les liaisons dangereuses

8. J. Laplanche, Sexual. La sexualité élargie au sens freudien, Paris, Puf, p. 231.

9. S. Freud, Psychopathologie de la vie quotidienne, Paris, Gallimard, 1997, p. 267.

10. S. Freud, « Leçons d'introduction à la psychanalyse », dans Euvres complètes, t. XIV, Paris, Puf, 2000, p. 99.

11. S. Freud, " Nouvelle suite des leçons d'introduction à la psychanalyse », dans Euvres complètes, t. XIX, Paris, Puf, 1995, p. 255.

12. « Leçons d'introduction... », op. cit., p. 21.

13. Ibid., p. 148-149. 
tributaires, à plus ou moins grande échelle, de ce que Freud nomme des « illusions », lesquelles consistent en une « surestimation de l'enchantement du mot, [en] la croyance que les processus réels du monde suivent les voies que notre pensée entend leur assigner ${ }^{14} »$. Dans ces modes de pensée à tendance illusoire, le désir prime sur la reconnaissance de la réalité (on y prend facilement ses désirs pour des réalités). Dans les modes de pensée scientifique, le désir de reconnaître la réalité prime sur les autres :

«La science est justement le plus parfait désengagement d'avec le principe de plaisir qui soit accessible à notre travail psychique ${ }^{15}$. »

Ce renoncement au plaisir de l'illusion est un long travail psychique ; il n'est pas donné chez l'homme, et encore ne s'accomplit-il jamais parfaitement :

«L'appareil psychique ne supporte pas le déplaisir, il lui faut s'en défendre à tout prix, et lorsque la perception de la réalité apporte du déplaisir, elle - c'est-à-dire la vérité - doit être sacrifiée ${ }^{16}$. »

La science est donc ce champ de production des discours le plus capable de renoncer à ses idées (ses croyances), même si cela cause du déplaisir, car le plaisir qui surclasse tous les autres - et qui est l'effet d'une discipline scientifique - est celui d'une reconnaissance des réalités. Ce sont donc les résultats d'expérimentations qui ont l'autorité la plus effective, plutôt que les plaisirs de la spéculation, de l'érudition, ou les conforts de la tradition :

«Les idées ne sont pas au fondement de la science, sur lequel tout repose ; ce fondement, au contraire, c'est l'observation seule. Ces idées ne sont pas le soubassement mais le faîte de tout l'édifice, et elles peuvent sans dommage être remplacées et enlevées ${ }^{17}$. »

Avant leur travail de scientifisation, les savoirs anciens sur les chiffres, les figures, le corps, l'histoire, la psyché, produisaient des vérités (mythologiques, religieuses, philosophiques). Lorsqu'ils se sont imposé les contraintes d'une recherche scientifique, ces champs de savoir ont ensuite requalifié leurs vérités premières d'«illusions ».

\section{Vérité, réalité, réel : des repères opératoires}

14. S. Freud, " Nouvelle suite des leçons... », op. cit., p. 250 .

15. S. Freud, « D'un type particulier de choix d'objet chez l'homme », dans S. Freud, Euvres complètes, t. X, Paris, Puf, 1993, p. 191. 16. S. Freud, « L'analyse finie et l'analyse infinie », dans Euvres complètes, t. XX, Paris, Puf, 2010, p. 38 .

17. S. Freud, « Pour introduire le narcissisme », dans Euvres complètes, t. XII, Paris, Puf, 2005, p. 221.

18. G. Bachelard, op. cit., p. 75 .
Qu'entend Freud par « vérité » et « réalité » ? Il ne s'agit pas ici de coller aux mots et de les prendre, sans critique, pour des choses. Les mots comme les idées ont des histoires. Celle de la notion de « vérité » serait à faire. Reste que Freud se situe dans le régime scientifique - occidental et moderne - de la vérité, devenu lui-même, avec ses propres critères, une tradition singulière. «Vérité » vient chez Freud qualifier des fragments de discours produits à partir d'une méthode et d'un dispositif scientifiques, faisant consensus en ce qu'ils rendent le mieux compte des phénomènes expérimentés. Les vérités scientifiques sont locales, fragiles et indexées à un mode de production ; elles permettent la localisation dans le discours d'un $x$ hors discours qu'elles constituent dès lors en réalité scientifique, jusqu'à ce que la reconnaissance d'un nouvel $x$ les mette à mal. Ces réalités scientifiques sont des constructions extraites de la réalité empirique ; elles appartiennent à ce que Bachelard nomme la « phénoménotechnique ${ }^{18} »$.

Il faut ici introduire un troisième terme freudien - le réel - pour saisir les deux autres. « Réel » est le mot qu'il utilise, comme les scientifiques de son temps, pour désigner l' $x$ inconnaissable au cœur des réalités scientifiques. La 
science part toujours de la croyance dans les réalités qu'elle a construites, mais de nouvelles expériences font émerger de nouveaux phénomènes, qui falsifient ces réalités, et donc les vérités discursives qui leur étaient attachées, que Kuhn a nommées les crises scientifiques. Il s'agit alors d'inventer un cadre nouveau où le « réel » puisse se résorber en réalité, dans le discours. De nouvelles vérités sont l'objet de créances, en un procès sans fin, raillé par les religions (aux vérités plus stables, puisque liées à l'interprétation de seuls textes) :

« [La religion dit de la science :] Tout ce qu'elle enseigne ne vaut que provisoirement ; ce que l'on prône aujourd'hui comme sagesse suprême sera rejeté demain et remplacé, de nouveau, à titre d'essai uniquement, par autre chose. La dernière erreur s'appelle alors vérité. Et c'est à cette vérité que nous sacrifierons notre bien suprême ${ }^{19}$ ! »

Il y a toute l'histoire de la philosophie derrière ce concept de « réel ». Au $\mathrm{XIX}^{\mathrm{e}}$ siècle, il est repris par le champ scientifique. Emil Du Bois-Reymond dira dans une conférence «Ignoramus. Ignorabimus ! : nous ignorons et ignorerons à jamais ${ }^{20}$. Ce qui est au cœur du mouvement scientifique, c'est donc la reconnaissance sans cesse relancée du réel. Celui-ci n'a pas d'unité, pas d'être (car il n'y a d'être que de langage). Il est un opérateur du repérage d'un « ça existe », à la limite et hors du discours. Il désigne le point d'ignorance, d'impossible savoir.

Freud revient tout au long de son œuvre sur ce repérage du réel, que Lacan remettra au centre de la pratique et de la théorie analytiques. À la toute fin de sa vie, dans l'Abrégé de psychanalyse (1940), il affirme encore : « Le réel restera toujours "inconnaissable ${ }^{21}$ ". »

\section{Scientificité de la psychanalyse}

\section{La pluralité des vérités et des sciences}

Les sciences déconstruisent la réalité commune, illusoirement unitaire. À mesure que sont reconnues des énigmes, de nouveaux cadres d'expérimentation sont inventés, lesquels disséminent du réel ${ }^{22}$. Il apparaît aux scientifiques qu'il y a autant de sciences que de lignes de fuite du réel. Et l'idéal unitaire, dont on peut supposer qu'il soit un désir partagé, est sans cesse ajourné, dans le champ scientifique, au profit du désir de vérité. Des réels existent, appelant des vérités plus larges ou contradictoires. Le champ scientifique conserve un idéal d'unité des savoirs ; mais il ne le fait pas primer sur le désir de vérité, sur la reconnaissance d'un réel. Il n'y a pas de « rebut du monde des phénomènes ${ }^{23}$ » pour la science. Un «ça existe » qui ne peut être relié à un champ d'objet inaugure l'invention d'un nouveau sous-champ. Ceux-ci naissent et disparaissent au cours de controverses.

Dès lors, on constate une pluralisation des dispositifs, des méthodes, des vérités et des sciences. Mais Freud constate que cette dissémination épistémologique déplaît à l'appareil psychique, lequel aspire à la synthèse, quitte à verser dans l'illusion, en occultant des fragments de réel :

«Dans les affaires scientifiques, on aime bien extraire une part de la vérité, la mettre à la place du tout, pour ensuite combattre en sa faveur tout le reste, qui n'est pas moins $\mathrm{vrai}^{24}$.»
Psychanalyse et science : les liaisons dangereuses

19. S. Freud, « Nouvelle suite des leçons... », op. cit., p. 257-258.

20. Sur ce point et pour une étude détaillée du champ des savoirs au temps de Freud, voir P.-L. Assoun, Introduction à l'épistémologie freudienne, Paris, Payot, 1981.

21. S. Freud, « Abrégé de psychanalyse », dans S. Freud, Euvres complètes, t. XX, op. cit., p. 294.

22. Voir S. Firestein, op . cit. 23. S. Freud, « Leçons d'introduction...», op. cit., p. 20. 24. Ibid., p. 359. 
« J'ai une forte aversion pour des simplifications faites aux dépens de la vérité, et je ne vois pas d'inconvénient à ce que vous ayez pleinement l'impression de la complexité et de l'enchevêtrement du sujet ${ }^{25}$. »

Lorsque le désir d'unité prime sur le désir de vérité, on sort de la science au profit de ce que Freud nomme des « visions du monde » scientifiques (Canguilhem les appelle « idéologies scientifiques $\left.{ }^{26} »\right)$. Ces visions unitaristes consistent à nier arbitrairement la multiplicité des champs d'objets. Tel était le scientisme du temps de Freud, dont le programme était une réduction de la réalité scientifique supposée « une » à l'ordre physico-chimique. Freud définit ainsi les visions du monde (qui peuvent être aussi bien politiques, religieuses, philosophiques que scientifiques) :

« J'estime donc qu'une vision du monde est une construction intellectuelle qui résout de façon unitaire tous les problèmes de notre existence à partir d'une hypothèse subsumante, dans laquelle par conséquent aucune question ne reste ouverte, et où tout ce qui retient notre intérêt trouve sa place déterminée. Il est aisé de comprendre que la possession d'une telle vision du monde est l'un des souhaits idéaux des hommes ${ }^{27}$. »

Le scientisme - qu'il soit mécaniciste, physicaliste, biologiste -, même s'il renaît régulièrement de ses cendres (cf. la psychiatrie biologique contemporaine), échoue manifestement à rendre compte de la complexité des faits. « La » science n'existe pas. L'histoire des sciences en témoigne. Aucun discours n'a encore pu produire une articulation totale de la réalité en ses divers aspects, en épuisant tous ses points de réel. Le champ scientifique s'est scindé en mathématiques, sciences physiques, sciences de la vie, puis sciences sociales. Les sciences physiques se sont elles-mêmes divisées en science classique et science quantique. Les sciences de la vie regroupent l'écologie, l'évolution, la génétique, la physiologie, l'anatomie, la zoologie, la botanique, la biochimie, et d'autres encore. Les sciences sociales sont pléthore.

De plus, toutes ces sciences ont des méthodes et des résultats différents. C'est pourquoi, pour Freud, c'est bien le travail de scientifisation et non son résultat qui fait la scientificité. Le résultat dépend du désir, de la méthode et de la nature de l'objet. La physique arrive à faire des prédictions dans le monde classique, car les objets y sont d'une complexité saisissable ; dans le monde quantique, cela n'est déjà plus possible. Dans la théorie évolutionniste, la sélection naturelle n'est qu'une hypothèse jamais observée, non testable, mais qui permet de rendre intelligible l'histoire naturelle. On ne sait toujours pas expliquer ni prévoir les mutations génétiques. En astronomie, on observe mais on ne peut expérimenter. La sociologie, l'archéologie, la linguistique et d'autres encore, proposent des dispositifs, des méthodes et des degrés de formalisation différents.

En somme, pour Freud, chaque science n'est légitime que pour un aspect

25. Ibid., p. 292.

26. G. Canguilhem, « Qu'estce qu'une idéologie scientifique ? », dans Idéologie et rationalité..., op. cit., p. 39-55.

27. S. Freud, « Nouvelle suite des leçons... », op. cit., p. 242. de «la réalité ». Les méthodes en anatomo-pathologie ne servent à rien lorsqu'il s'agit de déterminer la vitesse d'une particule quantique ; autres objets, autres dispositifs, autres méthodes. Et il n'est pas scientifique - mais idéologique d'appliquer de force la méthode inventée pour un champ d'objets à un autre :

«En soi, toute science est en effet unilatérale, elle l'est forcément, du fait qu'elle se restreint à des contenus, points de vue et méthodes déterminés. C'est un contresens auquel je ne voudrais avoir aucune part que de jouer une science contre 
une autre. La physique, après tout, n'enlève rien de sa valeur à la chimie, elle ne peut la remplacer ni non plus être représentée par elle. La psychanalyse est [tout autant] unilatérale, en tant qu'elle est la science de l'inconscient animique ${ }^{28}$.»

\section{La psychanalyse, une science parmi d'autres}

Freud désigne de façon précise le point de départ de l'invention de la psychanalyse : c'est pour lui le repérage du « réel » des symptômes hystériques, chez Charcot, via le concept paradoxal de « lésion dynamique ${ }^{29} »$. Ces symptômes font réel en ce qu'ils pointent une énigme. Comme le dit un jour Charcot à Freud, même si ces paralysies sont inexplicables et théoriquement impossibles, « ça n'empêche pas d'exister ${ }^{30}$ ». C'est la reconnaissance de ce réel qui provoque son activité théorique, le conduit à inventer un nouveau dispositif (fauteuil/divan) et une méthode inédite (associations libres de la parole), par où ce «ça existe » puisse être pratiqué et pensé. Qu'il y ait, au cœur de la pratique et de la théorie analytiques, un réel à penser, c'est ce dont Freud ne doute pas :

«L'inconscient est le psychisme proprement réel, aussi inconnu de nous dans sa nature interne que le réel du monde extérieur, et qui nous est livré par les données de la conscience tout aussi incomplètement que l'est le monde extérieur par les indications de nos organes sensoriels ${ }^{31}$. »

« C'est avec un haussement d'épaules résigné que nous devons repousser comme incompréhensible ce qu'on nous objecterait, à savoir que l'inconscient n'est ici rien de réel au sens de la science, qu'il est un expédient de fortune, une façon de parler. Quelque chose de non réel, dont procèdent des effets aussi réellement saisissables qu'une action de contrainte ${ }^{32}$ ! »

De la même manière qu'un jour la biologie s'est détachée de la physique pour penser le réel du vivant, au cœur de la matière inerte, la psychanalyse doit se détacher de la biologie pour penser le réel du vivant parlant, au cœur de la matière vivante :

« Sa contribution à la science [Freud parle de la psychanalyse] consiste justement dans l'extension de la recherche au domaine animique. Sans une telle psychologie, la science serait, il est vrai, très incomplète ${ }^{33}$.»

L'édifice psychanalytique ne tient que par cette orientation sur le réel d'une incidence du langage sur le corps, point aveugle de la biologie moderne, empiriquement pratiquée par de multiples traditions psychothérapeutiques, mais scientifiquement théorisée - et par là nouvellement pratiquée - par la psychanalyse. Freud peut en conclure que la psychanalyse est une conséquence du désir de vérité scientifique :

«Il ne faut pas oublier que la relation analytique est fondée sur l'amour de la vérité, c'est-à-dire sur la reconnaissance de la réalité et qu'elle exclut tout semblant et tout leurre ${ }^{34}$.»

La psychanalyse ne produit pas ses réalités par simple performance discursive, comme les mythologies, les religions, les philosophies, l'art ou la littérature. Elle repère et tente de rendre intelligible un réel (en construisant l'appareil psychique, ses pulsions et représentations) pour opérer sur celui-ci. Ainsi dans son versant théorique, la psychanalyse est-elle une science parmi d'autres :

«La psychologie est [...] une science de la nature. Que pourrait-elle donc être d'autre $^{35}$ ?»
Psychanalyse et science : les liaisons dangereuses
28. S. Freud, « La question de l'analyse profane », dans Euvres complètes, t. XVIII, Paris, Puf, 1994, p. 57.

29. S. Freud, Contribution à la conception des aphasies (1891), Paris, Puf, 1983, p. 54 .

30. S. Freud, « Autoprésentation », dans Euvres complètes, t. XVII, Paris, Puf, 1992, p. 61.

31. S. Freud, « L'interprétation du rêve », dans CEuvres complètes, t. IV, Paris, Puf, 2004, p. 668.

32. S. Freud, « Leçons d'introduction à la psychanalyse ", dans Euvres complètes, t. XIV, op. cit., p. 247. 33. S. Freud, " Nouvelle suite des leçons... », op. cit., p. 243-244.

34. S. Freud, "L'analyse finie et l'analyse infinie », $o p$. cit., p. 50.

35. « Some elementary lessons in psycho-analysis » (1938), dans S. Freud, Euvres complètes, t. XX, op. cit., p. 311. 
Dans son versant clinique, le repérage des vérités devient le moyen même par lequel le soin s'effectue, au cœur d'une rencontre extrascientifique entre deux vivants parlants. L'insupportable réel (pulsionnel), dès lors qu'il est élaboré dans le discours par un patient, permet des transformations dans la vie psychique, et un passage du statut de «patient » à celui d' « analysant », signé par une mise au travail propre du sujet :

«Si la construction [proposée au patient] est fausse, rien n'est changé chez le patient ; mais si elle est exacte ou si elle représente un pas vers la vérité, il y réagit $^{36}$.»

Par où l'on voit qu'il n'y a de réel - et d'effets liés à son existence - qu'à l'intérieur d'un dispositif et d'une méthode. Celui de la pratique analytique est lié à la parole. Les fragments de réel, ainsi localisés par des représentations, prennent le nom scientifique de «vérité[s] historique $[\mathrm{s}]^{37}$ », propres au sujet; il s'agit qu'elles soient énoncées et validées.

Dans les Études sur l'hystérie, Freud traque des fragments de réel propres à chacune de ses patientes. Il peut s'en dire quelque " chose », jusqu'à un point d'insaisissable, qu'il nomme, dans L'interprétation du rêve, « l'ombilic $[\ldots]$, le point où [une représentation] repose sur le non connu ${ }^{38} »$. Cet ombilic psychique est ce qu'il borde du nom de pulsion : la pulsion investit la représentation, par où elle est saisissable, mais se perd également dans le bios le plus opaque, en direction de ce que les biologistes nomment «l'excitation ». C'est sa part de « reste non résolu ${ }^{39}$ ».

Le réel analytique - à saisir comme point de jonction du vivant et du langage - se manifeste soit (délié) sous forme d'angoisse ou de compulsion de répétition, soit (lié) sous forme de plaisir ou de déplaisir. D'arpenter ce réel par la parole a des effets sur le corps du vivant parlant ; c'est là l'énigme avec laquelle travaille la psychanalyse, sans pouvoir la résoudre entièrement.

Le réel de la théorie analytique est une extension de celui de la pratique clinique, en tant qu'il provoque la pensée. À partir de l'expérience de ces fragments de réel singuliers, Freud a construit un savoir général, minimum, de quelques concepts tout au plus : la métapsychologie (cf. les concepts de pulsion, représentation, processus primaires et secondaires, mécanismes de défense, transfert, etc.). Elle est cette science pauvre lui permettant de se repérer dans la parole analysante pour y opérer. Cette science, comme les autres, est « open to revision ${ }^{40} »$.

36. S. Freud, « Construction dans l'analyse », dans Euvres complètes, t. XX, op. cit., p. 69.

37. S. Freud, « Un souvenir d'enfance de Léonard de Vinci », dans Euvres complètes, t. X, op. cit., p. 109 38. S. Freud, « L'interprétation du rêve », op. cit., p. 578. 39. Ibid., p. 435.

40. S. Freud, « La question de l'analyse profane », op. cit., p. 17.

41. S. Freud, « Inhibition, symptôme et angoisse », dans Euvres complètes, t. XVII, op. cit., p. 241.
La psychanalyse a enfin pour conséquence de repenser l'activité scientifique même, en repérant un réel intraitable (la pulsion), interdisant, de structure, l'accomplissement du programme scientifique de réduction du réel au discours. Elle pointe la part mythique (illusoire) de toute activité scientifique. Sans dévaluer le désir scientifique de savoir, elle en établit méta-psychologiquement (scientifiquement) les limites :

«Si nous ne pouvons voir clair, du moins voulons-nous voir clairement les obscurités ${ }^{41}$.»

\section{Conclusion}

Freud montre qu'il n'y a pas de science idéale qui puisse donner aux autres ses critères de scientificité - comme le croira Popper, à partir d'une idéalisation des sciences physiques et de ses critères (haut degré de formalisation théorique, 
falsifiabilité, universalité, prédictibilité des phénomènes) -, mais des sciences hétérogènes, unies par un même esprit : une éthique de la vérité scientifique. Au début, l'idéal scientiste d'une science unique le fait encore douter de la scientificité de la psychanalyse :

« Je n'ai pas toujours été psychothérapeute, mais comme d'autres neuropathologistes, j'ai été formé aux diagnostics locaux et au pronostic électrique, et cela ne cesse de me faire à moi-même une impression singulière de voir que les histoires de malades que j'écris se lisent comme des nouvelles et sont pour ainsi dire privées de l'empreinte de sérieux de la scientificité. Je dois m'en consoler en me disant que c'est évidemment la nature de l'objet qui doit être rendue responsable de ce résultat, bien plus que mes prédilections ${ }^{42}$. »

Mais son expérience l'amène à reconnaître que, même si la clinique était idéologiquement mise en équations (comme dans les sciences physiques), elle n'en serait pas pour autant plus vraie. Au contraire, car c'est la logique de l'objet, non l'idéalisation d'un résultat, qui guide le travail scientifique. Ainsi Freud, trente ans après avoir vu ses premières réflexions clinique être taxées de « conte scientifique ${ }^{43}$ » par Krafft-Ebbing, renvoie-t-il la critique à l'envoyeur :

«Il me fallut malheureusement faire bientôt l'expérience qu'en suivant ses prescriptions [celles de Wilhelm Erb, à propos des paralysies hystériques] on n'arrivait à rien, que ce que j'avais tenu pour le précipité d'une observation exacte n'était qu'une construction de la fantaisie [et] n'avait pas plus de relation à la réalité que, par exemple, un livre des rêves "égyptien ${ }^{44 "}$ " »

Dès lors qu'il s'agit de repérer et d'opérer au mieux sur le réel qui surgit au détour du dispositif analytique, dans le transfert, la psychanalyse reste à ce jour l'approche la plus adéquate - la plus vraie - dans son champ d'objet et, à ce titre, jusqu'à preuve du contraire, la plus scientifique. Qu'elle soit scientifique ne signifie cependant pas - c'est justement ce dont elle fait science qu'elle soit toute scientifique. Reste une part qui ne l'est pas :

«Ce que j'ai dit est la vérité, pas toute la vérité, certes, mais une part très considérable de celle-ci ${ }^{45}$. »

Ce retour à l'épistémologie freudienne nous aura enseigné une chose : le transfert à Freud, à bien lire ses textes, doit se faire sur son désir de vérité plus que sur les concrétions textuelles laissées derrière lui ; suivre Freud, c'est, en prenant appui sur l'œuvre, viser au-delà : en direction du réel clinique auquel sa pratique et sa pensée ont tenté de répondre.

Qu'en est-il, aujourd'hui dans notre champ, de cette orientation scientifique par le réel exigée par Freud ? En lui redonnant du crédit, on saurait prendre nos distances avec les excès transférentiels, la fétichisation des textes et les illusions de groupe. Le discours analytique affinerait son statut épistémologique, se resserrant sur ce qui opère cliniquement, renonçant à cette inflation d'érudition qui mêle trop souvent - en d'obscures jouissances du sens - clinique, poésie, littérature et philosophie. Il se libérerait de ses interdits de pensée, d'évidences sacrées, pour renouer avec le courage scientifique de l'ignorance.

N'est-il pas temps d'envisager que, pour redonner légitimité et avenir à notre discipline, une refondation du champ sur le désir scientifique de vérité serait opportune, qui le soumettrait à plus d'empiricité, de critique - intra et interdisciplinaire - et de réflexivité ?
Psychanalyse et science : les liaisons dangereuses
42. S. Freud, «Études sur l'hystérie ", dans Euvres complètes, t. II, Paris, Puf, 2009, p. 182.

43. Sigmund Freud. Lettres à Wilhelm Fliess. 1887-1904, Paris, Puf, 2006, p. 236 (lettre à Fliess du 26 avril 1896).

44. S. Freud, « Autoprésentation », op. cit., p. 63-65.

45. S. Freud, « La question de l'analyse profane », op. cit., p. 46. 


\section{Le Coq-Héron 222 Résumé}

Le débat sur la scientificité de la psychanalyse est récurrent depuis Freud. Mais la façon dont on le pose ne conduit-elle pas à une impasse ? La psychanalyse n'aurait d'autre alternative qu'être une science impossible ou une nécessaire anti-science. L'auteur tente ici, en s'appuyant sur les réflexions épistémologiques de Freud, de redonner sa place à une autre conception du travail scientifique, quitte à décaler le débat. À désidéaliser « la » science, à reconnaître la pluralité des constructions d'objet, méthodes et dispositifs scientifiques, on se permet de concevoir la psychanalyse comme une science parmi d'autres, avec son objet (le réel pulsionnel) et ses propres seuils de formalisation.

\section{Mots-clés}

Psychanalyse, science, scientisme, vérité, réel, épistémologie, pluralisme, religion, foi. 